Research Article

\title{
Prevalence and Associated Factors of Chronic Kidney Disease among Adult Hypertensive Patients at Northwest Amhara Referral Hospitals, Northwest Ethiopia, 2020
}

\author{
Anteneh Hunegnaw, ${ }^{1}$ Habtamu Sewunet Mekonnen, ${ }^{2}$ Masresha Asmare Techane, ${ }^{3}$ \\ and Chilot Desta Agegnehu $\mathbb{1}^{4}$ \\ ${ }^{1}$ University of Gondar, Comprehensive Specialised Hospital, Gondar, Ethiopia \\ ${ }^{2}$ Department of Medical Nursing, School of Nursing, College of Medicine and Health Sciences, University of Gondar, \\ Gondar, Ethiopia \\ ${ }^{3}$ Department of Pediatrics and Child Health Nursing, School of Nursing, College of Medicine and Health Sciences, \\ University of Gondar, Gondar, Ethiopia \\ ${ }^{4}$ School of Nursing, College of Medicine and Health Sciences and Comprehensive Specialized Hospital, University of Gondar, \\ Gondar, Ethiopia
}

Correspondence should be addressed to Chilot Desta Agegnehu; chilotdesta@gmail.com

Received 30 January 2021; Revised 2 April 2021; Accepted 8 August 2021; Published 27 August 2021

Academic Editor: Thereza Maria Magalhães Moreira

Copyright (c) 2021 Anteneh Hunegnaw et al. This is an open access article distributed under the Creative Commons Attribution License, which permits unrestricted use, distribution, and reproduction in any medium, provided the original work is properly cited.

\begin{abstract}
Background. Chronic kidney disease (CKD) is a progressive loss of the kidney function which leads to a decreased kidneys' ability to process waste in the blood and it affects the other important functions of the kidney. The disease has different stages that can alter the health status of individuals. During the early stages, patients may present with a normal or slight decrease in glomerular filtration rate (GFR) and albuminuria. Later, it progresses and leads to end-stage renal disease (ESRD) or kidney failure. Hypertension is considered as the major contributing risk factor of CKD. Objective. This study was aimed to assess the prevalence and associated factors of chronic kidney disease among adult hypertensive patients in referral hospitals of the Northwest Ethiopia. Methods. An institution-based cross-sectional study was conducted among 581 adult hypertensive patients in a chronic follow-up clinic in referral hospitals, Northwest Ethiopia, from July to August 2020. Systematic random sampling was used to select the study participants. Data were collected using the interviewer-administered questionnaire and participants medical records. Both bivariable and multiple logistic regression analyses were performed. Model fitness was assessed using a Hosmer-Lemeshow test. Result. The total prevalence of CKD among adult hypertensive patients was 17.6\% (95\% CI: 14.7-20.8). Diastolic blood pressure $\geq 90 \mathrm{mmHg}(\mathrm{AOR}=8.65 ; 95 \% \mathrm{CI}: 4.77-15.68)$, duration of hypertension $\geq 10$ years (AOR $=8.81 ; 95 \% \mathrm{CI}: 2.47-31.45)$, stage II HTN $(\mathrm{AOR}=2.61 ; 95 \% \mathrm{CI}: 1.04-6.50)$, comorbid disease $(\mathrm{AOR}=7.0 ; 95 \% \mathrm{CI}: 2.20-22.21)$, proteinuria $(\mathrm{AOR}=4.59 ; 95 \% \mathrm{CI}$ : 2.08-10.12), dyslipidemia ( $\mathrm{AOR}=3.40 ; 95 \% \mathrm{CI}: 1.56-7.24)$, and serum creatinine $\geq 1 \mathrm{mg} / \mathrm{dl}$ (AOR = 8.88; 95\% CI: 4.40-17.91) were associated with chronic kidney disease among adult hypertensive patients. Conclusion. In this study, the prevalence of CKD among hypertensive patients found was $17.6 \%$. Regarding associated factors, dyslipidemia, proteinuria, comorbid disease, serum creatinine greater than $0.9 \mathrm{mg} / \mathrm{dl}$, duration of hypertension greater than 10 years, and diastolic blood pressure greater than $90 \mathrm{mmHg}$ are factors associated with the occurrence of chronic kidney disease among hypertensive patients.
\end{abstract}

\section{Background}

Chronic kidney disease (CKD) can be explained as a progressive loss of kidney function which results in decreased kidneys' ability to process waste and it affects the other important functions of the kidney [1]. The disease has different stages that can alter the health status of individuals. During the early stages, patients may present with a normal or slight decrease in glomerular filtration rate (GFR) and albuminuria. Later, it progresses and leads to end-stage renal 
disease (ESRD) or kidney failure [2-4]. Eventhough, the etiology of CKD still needs investigation [5]. Hypertension is considered as the major contributing risk factor of CKD $[6,7]$.

CKD usually gets worse over time though treatment has been shown to slow progression. If left untreated, it can progress to kidney failure and early cardiovascular disease. When the kidneys stop working, dialysis or kidney transplant is needed for survival. These would have negative impacts and burdens on patients, their families, and healthcare setups at large to the countries $[8,9]$.

Globally, the prevalence of CKD is significantly rising, and it is associated with major morbidity and mortality that demands special attention as one of the growing public health problems [10]. As it is evidenced by studies in the general population, the prevalence of CKD was $13.4 \%$ [10], while the pooled prevalence of CKD in patients with hypertensive was recorded higher as compared to the general population which is $24.7 \%$ [11]. Furthermore, as hypertension is the leading risk factor and causes for chronic kidney disease, the prevalence was higher compared with the general population [12].

CKD is associated with an increase in CVD and premature mortality, with detrimental societal and economic challenges $[13,14]$.

In 2017, CKD was the 12th leading cause of death worldwide and it resulted 1.2 million deaths, Besides, $7.6 \%$ of all CVD deaths (1.4 million) attributed to impaired kidney function. Deaths due to CKD or to CKD-attributable CVD accounted for $4.6 \%$ of all-cause mortality [15].

As the global burden of disease 2015 study, CKD was the leading cause of death and one of the fastest rising major causes of deaths in which overall mortality due to CKD has increased by $31.7 \%$ from 2005 to 2015 [16]. Moreover, in lower and middle-income countries, the prevalence is estimated to be $15 \%$ higher compared with high-income countries [17].

The prevalence of CKD among the general population in India, Korea, Pakistan, and Southern China were $17.2 \%$, $13.7 \%, 12.5 \%$, and $9.4 \%$, respectively $[6,18,19]$.

A systematic review in Africa continent indicates that the prevalence of CKD differs from $2 \%$ to $41 \%$, and the prevalence among hypertensive patients is ranging from $13 \%-$ $51 \%$. This systematic review showed that the prevalence of CKD in Burundi, Mali, Nigeria, Egypt, Seychelles, Uganda, Congo, Kenya, Ghana, Cameroon, South Africa, Tanzania, Sudan, Senegal, and Morocco was 45.7\%, 40.8\%, 29.7\%, 18\%, $15.3 \%, 15.2 \%, 12.4 \%, 11.5 \%, 10.2 \%, 10 \%, 7.4 \%, 7 \%, 6.4 \%$, $6.1 \%$, and $2.9 \%$, respectively [20].

A cross-sectional study conducted in Ethiopia at Tigray teaching hospital and Jimma University both indicate that the prevalence of CKD among hypertensive patients was $22.1 \%$ and $26 \%$, respectively $[21,22]$.

As studies showed in the Glob, age, sex, educational status, residence, marital status, body mass index, comorbidity, type of antihypertensive medications, smoking, alcohol intake, and sedentary lifestyle were the associated factors with CKD [11, 18, 19, 21-28].
The World Health Organization (WHO) report, Ethiopia 2018 , indicates that more than $39 \%$ of yearly deaths were recorded as a result of the noncommunicable disease including $\mathrm{CKD}$ and hypertension [12]. Additionally, CKD has a devastating effect on the nation's health sector and economy. However, very little is known about it in the study setting. Therefore, this study was aimed to assess the prevalence and associated factors of CKD among hypertensive patients.

\section{Methods and Materials}

2.1. Study Design and Period. An institution-based crosssectional study was conducted from July to August/2020.

2.2. Study Area. The study was conducted in Northwest Ethiopia referral hospitals, namely, the University of Gondar Teaching Referral Hospital (UOGTRH), Felege Hiwot Referral Hospital (FHRH), and Debre Markos Referral Hospital (DMRH).

Each of them were estimated to serve for 5-7 million peoples in their catchment area, and UOGTRH has 1200 hypertensive patients, FHRH has 800 hypertensive patients, and DMRH has 600 hypertensive patients, which makes a total of 2600 hypertensive patients attending the chronic illness followup clinic in these catchment areas. UOGTRH is found in the Northern part of Amhara Region in Gondar town which has 9 nurses and 4 doctors who work in the chronic illness follow-up clinic regularly. FHRH is found in Bahirdar city, which is the capital city of Amhara Region, which has 8 nurses who work in the clinic regularly, and DMRH is again found at the east of Gojam in Amhara regional state, Northwest Ethiopia, which has 8 nurses who work in the clinic regularly.

2.3. Source and Study Populations. All hypertensive patients aged 18 years and above who had follow-up at the chronic illness clinic of referral hospitals in Northwest Ethiopia were the source population and those who attended during the data collection were the study population.

2.4. Inclusion Criteria. All adult hypertensive patients who had hypertension treatment follow-up at the three referral hospitals and who had medical records.

2.5. Exclusion Criteria. Pregnant women who were diagnosed with pregnancy-induced hypertension were excluded.

\subsection{Sample Size Determination and Sampling Procedure}

2.6.1. Sample Size Determination. To get the adequate sample size, both the single population proportion formula and significantly associated factors from other study were computed (Table 1).

Accordingly, a single population proportion formula was calculated considering the following assumptions: 95\% confidence interval (CI), $22.1 \%$ population proportion from the previous study [26], and $4 \%$ marginal error. 
TABLe 1: Sample size calculation using associated factors.

\begin{tabular}{lccc}
\hline No. & Associated factors & Assumption & Number \\
\hline 1 & BMI & OR $=1.38[29], \%=32 \%, p=80, R=1$ & 528 \\
2 & Body swelling & OR=3.01[29], \%=32\%,p=80, $R=1$ & 122 \\
3 & Uncontrolled BP & OR=2.92[29], \%=25, $p=80, R=1$ & 284 \\
\hline
\end{tabular}

$$
n=\frac{(Z(\alpha / 2)) 2 * p(1-p)}{(d) 2}
$$

$n$ represents the initial sample size; $Z \alpha / 2$ represents the standardized normal distribution value for the $95 \%$ $\mathrm{CI}=1.96 ; P$ represents the proportion of chronic kidney disease $(22.1 \%)$; $d$ represents the margin of error $4 \%$.

$$
N=\frac{(1.96)^{2} \times 0.221(1-0.221)}{(0.04)^{2}}=413 \text {. }
$$

By considering a $10 \%$ nonresponse rate, the sample size was $413+41=454$.

By considering a $10 \%$ nonresponse rate, the final sample size was $528+53=581$. Since the largest sample size (581) was found in associated factors, it was taken as the final sample size.

2.6.2. Sampling Procedure. The total of 581 sample populations were proportionally allocated to each referral hospitals. About 268, 179, and 134 participants were selected in the University of Gondar Comprehensive Specialized Hospital, Felege Hiwot Referral Hospital, and Debre Markos Referral Hospital, respectively. Finally, study participants were selected using systematic random sampling as per their coming order. To avoid the recycling of data, special marks were put in participants' medical charts.

\subsection{Variables of the Study}

Dependent variable

(i) Chronic kidney disease (yes/no)

Independent variables

(i) Sociodemographic characteristics: age, sex, religion, educational status, occupation, income, marital status, residence, and family history of hypertension.

(ii) Clinical characteristics: BP status, baseline systolic $\mathrm{BP}$, baseline diastolic BP, stage of hypertension, type of hypertension, type of drugs, duration of drugs, number of drugs, comorbidity, dyslipidemia, proteinuria, and obesity.

(iii) Lifestyle characteristics: smoking, alcohol use, and exercise.

\subsection{Operational Definition}

Chronic kidney disease: CKD is defined and identified as when physicians diagnosed the patient as already having chronic kidney disease [29].
Controlled blood pressure: $\mathrm{BP}<140 / 90 \mathrm{mmHg}$ in the last follow-up using a digital sphygmomanometer for adult hypertensive clients without diabetes mellitus and chronic kidney disease and blood pressure <130/ $80 \mathrm{mmHg}$ using a digital sphygmomanometer for adult hypertensive clients with diabetes mellitus and chronic kidney disease in the last follow-up record [29].

BMI (body mass index): A measure of the person's weight in kilogram divided by his height in meter square; based on this calculation, bodyweight was classified as underweight for a patient with less than $18.5 \mathrm{~kg} / \mathrm{m}^{2}$, normal weight $18.5-24.9 \mathrm{~kg} / \mathrm{m}^{2}$, overweight $25-29.9 \mathrm{~kg} / \mathrm{m}^{2}$, and obese for patients with $\mathrm{BMI} \geq 30 \mathrm{~kg} / \mathrm{m}^{2}$ [2]

Proteinuria: according to the laboratory result, if a patient had plus one and above, we classify as having proteinuria in the last laboratory result [29].

Hypertension: it was defined as those who had a documented diagnosis of hypertension (i.e., BP $\geq 140$ / $90 \mathrm{mmHg}$ ) or those on antihypertensive agents. Target control BP was defined as BP $<140 / 90 \mathrm{mmHg}$ for nondiabetic and $<130 / 80 \mathrm{mmHg}$ for diabetic patients [30].

Estimated GFR: this is a test that is used to assess how well kidneys are working. The test estimates the volume of blood that is filtered by the kidneys over a given period. It can be measured by using readily available equations and formulas like Cockcroft-Gault. Cockcroft-Gault formula (normalized for the body surface area (BSA)): $(140-$ age (years) $) \times$ weight $(\mathrm{kg}) \times(0.86$, if female $) \times 1.73 / 72 \times$ serum creatinine $(\mathrm{mg} / \mathrm{dL}) \times \mathrm{BSA}$ $\left(\mathrm{m}^{2}\right)$. In this case, BSA will be calculated by using the Mosteller formula, that is, BSA is equal to $\sqrt{W \times H}$ divided by 60 , while weight is measured in kilogram and height in centimeter [31].

Alcohol exposure: participant; intake of alcohol, according to the patient's record of taking any alcoholic drinks will be considered as alcohol consumer [21].

Smoking: the patient's response/record of who smokes or ever smoke [32].

Physical exercise: the patients say they do perform the daily active exercise for about 30 minutes a day [28].

Comorbidity: it is the presence of any health conditions other than hypertension.

2.9. Data Collection Tool and Procedure. A standardized tool was used for data extraction. The tool contains sociodemographic and baseline clinical and treatment-related variables. In addition to face-to-face interview, forms were 
used for laboratory request and follow-up records of hypertension, and patient cards were reviewed.

Two days training was given for data collectors regarding each description of the tool and the way of data collection from patients and their medical records using the extraction tool. For the sake of appropriate data collection, four nurses working in a chronic clinic or at least who have previous experience in an outpatient chronic clinic participated in data collection; one health officer has participated as a supervisor. Then, data were collected from the patient and their medical charts by using a prepared extraction tool. In this way, all patients who fulfill the inclusion criteria were interviewed, and data extraction was completed.

2.10. Data Quality Control. To maintain data quality, an appropriate and standardized extraction tool was used. The data extraction tool was pretested for consistency and completeness of data items on 5\% [25] of patient charts at the same hospital. Quality was also maintained by using experienced nurses in the chronic clinic. Even training was given to data collectors for one day before the data collection on the objective of the study and how to extract data for this study purpose using the data extraction format. Each component of the tool was discussed clearly for data collectors. The data extraction process was monitored closely by the supervisor throughout the data collection period. Data clerks also assist data collectors by identifying the charts. After filling each tool, completeness was checked, and the correction was made before the chart returned.

2.11. Data Processing and Analysis. Data were entered into Epi-Info version 7.2.2.6 and then transferred to SPSS version 23 for analysis. Summary statistics were used to look for any missing, and logistic regression was also used to determine crude and adjusted odds ratio (OR). Bivariate analysis was carried out to assess an association between the dependent and all the independent variables, and variables with a $p$ value $<0.2$ were further entered into the multivariable logistic regression model to control the possible effect of confounders. Model fitness was assessed using the Hosmer-Lemeshow test $(0.223)$. Odds ratios with $95 \%$ confidence interval at $p$ value $<0.05$, cleaning, and analysis were computed to determine the level of significance.

\section{Results}

3.1. Sociodemographic Characteristics of Participants. In this study, 581 patients were included with a response rate of $100 \%$. Of these, $55.1 \%$ were females, the median and standard deviation of the age was 54.71 and 11.397 , respectively, 476 (81.9\%) were married, 120 (20.7\%) had no formal education, $429(73.8 \%)$ were urban inhabitants, 497 (85.5\%) were orthodox followers, and 140 (24.1\%) were government employee (Table 2).

3.2. Clinical Related Characteristics. Of 581 study subjects, $64(11 \%)$ and $54(9.3 \%)$ of them have greater than and equal to $140 \mathrm{mmHg}$ for systolic and $90 \mathrm{mmHg}$ for diastolic blood
TABLE 2: Sociodemographic characteristics of hypertensive patients attending at the chronic follow-up clinic at Northwest Ethiopia referral hospitals, 2020.

\begin{tabular}{|c|c|c|c|}
\hline Variable & Category & Frequency & Percentage \\
\hline \multirow{2}{*}{ Sex } & Male & 261 & 44.9 \\
\hline & Female & 320 & 55.1 \\
\hline \multirow{2}{*}{ Age } & $<60$ years & 394 & 67.8 \\
\hline & $\geq 60$ years & 187 & 32.2 \\
\hline \multirow{4}{*}{ Marital status } & Single & 18 & 3.1 \\
\hline & Married & 476 & 81.9 \\
\hline & Divorced & 28 & 4.8 \\
\hline & Widowed & 59 & 10.2 \\
\hline \multirow{3}{*}{ Religion } & Orthodox & 497 & 85.5 \\
\hline & Muslim & 53 & 9.1 \\
\hline & Protestant & 31 & 5.3 \\
\hline \multirow{4}{*}{$\begin{array}{l}\text { Level of } \\
\text { education }\end{array}$} & No formal education & 120 & 20.7 \\
\hline & Primary & 124 & 21.3 \\
\hline & Secondary & 164 & 28.2 \\
\hline & Diploma and above & 173 & 29.8 \\
\hline \multirow{6}{*}{ Occupation } & $\begin{array}{l}\text { Government } \\
\text { employee }\end{array}$ & 140 & 24.1 \\
\hline & Farmer & 66 & 11.4 \\
\hline & Private & 92 & 15.8 \\
\hline & Merchant & 45 & 7.7 \\
\hline & Housewife & 159 & 27.4 \\
\hline & Others & 79 & 13.6 \\
\hline \multirow{2}{*}{ Residence } & Urban & 429 & 73.8 \\
\hline & Rural & 152 & 26.2 \\
\hline
\end{tabular}

*Others, retired and not yet working.

pressure, and $429(73.8 \%)$ of them have a BMI of 18.5-24.9. Also, 288 (49.6\%) of them are in the second stage of hypertension, 221 (38\%) of them have the comorbid disease, and $394(67.8 \%)$ of patients use calcium channel blockers for the treatment of hypertension (Table 3 ).

3.3. Lifestyle Factors. Among the 581 study subjects, 119 (20.5\%) of them are alcohol users and only 28 (4.8\%) of them are doing exercise on regular basis (Table 4).

3.3.1. Prevalence of Chronic Kidney Disease among Adult Hypertensive Patients. The total prevalence of CKD among hypertensive patients was $17.6 \%$ (95\% CI: 14.7-20.8).

3.3.2. Associated Factors of Chronic Kidney Disease among Adult Hypertensive Patients. In bivariable analysis, age less than 60 years, systolic blood pressure greater than $140 \mathrm{mmHg}$, diastolic blood pressure greater than $90 \mathrm{mmHg}$, stage II and III hypertension, duration of hypertension greater than 10 years, comorbid disease, drugs other than hypertension, proteinuria, dyslipidemia, serum creatinine, body swelling, beta-blocker, diuretics, and vasodilators, smoking, alcohol use, and exercise were associated with chronic kidney disease at $p$ value $<0.2$.

However, in multivariable analysis, DBP $\geq 90 \mathrm{mmHg}$, duration of hypertension $\geq 10$ years, stage II HTN, proteinuria, dyslipidemia, and serum creatinine $\geq 0.9 \mathrm{mg} / \mathrm{dl}$ 
TABLE 3: Clinical factors of hypertensive patients attending at the chronic follow-up clinic at Northwest Ethiopia referral hospitals.

\begin{tabular}{|c|c|c|c|}
\hline Factors & Category & Frequency & Percentage \\
\hline \multirow{2}{*}{ DBP } & $<90$ & 527 & 90.7 \\
\hline & $\geq 90$ & 54 & 9.3 \\
\hline \multirow{2}{*}{ SBP } & $<140$ & 517 & 89.0 \\
\hline & $\geq 140$ & 64 & 11.0 \\
\hline \multirow{2}{*}{ Duration of HTN (years) } & $<10$ & 550 & 94.7 \\
\hline & $\geq 10$ & 31 & 5.3 \\
\hline \multirow{4}{*}{ BMI } & $<18.5$ & 15 & 2.6 \\
\hline & $18.5-24.9$ & 429 & 73.8 \\
\hline & $25-29.9$ & 94 & 16.2 \\
\hline & $>30$ & 43 & 7.4 \\
\hline \multirow{3}{*}{ Stage of HTN } & Stage I & 262 & 45.1 \\
\hline & Stage II & 288 & 49.6 \\
\hline & Stage III & 31 & 5.3 \\
\hline \multirow{2}{*}{ Comorbid disease } & No & 360 & 62.0 \\
\hline & Yes & 221 & 38.0 \\
\hline \multirow{2}{*}{ Family Hx of HTN } & No & 541 & 93.1 \\
\hline & Yes & 40 & 6.9 \\
\hline \multirow{2}{*}{$\begin{array}{l}\text { Drugs other than anti-HTN } \\
\mathrm{Rx}\end{array}$} & No & 373 & 64.2 \\
\hline & Yes & 208 & 35.8 \\
\hline \multirow{2}{*}{ Proteinuria } & No & 488 & 84.0 \\
\hline & Yes & 93 & 16.0 \\
\hline \multirow{2}{*}{ Dyslipidemia } & No & 436 & 75.0 \\
\hline & Yes & 145 & 25.0 \\
\hline \multirow{2}{*}{ Body swelling } & No & 522 & 89.8 \\
\hline & Yes & 59 & 10.2 \\
\hline \multirow{2}{*}{ ACE inhibitor } & No & 297 & 51.1 \\
\hline & Yes & 284 & 48.9 \\
\hline \multirow{2}{*}{ Beta-blocker } & No & 525 & 90.4 \\
\hline & Yes & 56 & 9.6 \\
\hline \multirow{2}{*}{ Diuretics } & No & 329 & 56.6 \\
\hline & Yes & 252 & 43.4 \\
\hline \multirow{2}{*}{ Calcium channel blocker } & No & 187 & 32.2 \\
\hline & Yes & 394 & 67.8 \\
\hline \multirow{2}{*}{ Vasodilator } & No & 462 & 79.5 \\
\hline & Yes & 119 & 20.5 \\
\hline
\end{tabular}

were associated with chronic kidney disease among hypertensive patients at $p$ value $<0.05$.

The odds of CKD were 8.65 times (AOR $=8.65,95 \% \mathrm{CI}$ : 4.77-15.68) higher among those who had diastolic blood pressure greater than $90 \mathrm{mmHg}$ as compared to their counter parts.

Patients with duration of hypertension $\geq 10$ years were $(\mathrm{AOR}=8.81 ; 95 \%$ CI $2.47-31.45)$ more likely to develop $\mathrm{CKD}$ as compared to patients with duration of hypertension $<10$ years.

Being stage II HTN patients was $2.61(\mathrm{AOR}=2.61 ; 95 \%$ CI: 1.04-6.50) times higher among those patients at stage I HTN.

The odds of having $\mathrm{CKD}$ was 4.59 times $(\mathrm{AOR}=4.59 ; 95 \%$ CI: $2.08-10.12$ ) higher among proteinuria as compared to those patients free from proteinuria. Patients with the comorbid disease were 7 times (AOR $=7$; 95\% CI: 2.2-22.2) more likely to develop CKD as compared to their counter parts. Regarding to serum creatinine, patients who had
TABLE 4: Lifestyle factors of hypertensive patients attending at chronic follow-up clinic at Northwest Ethiopia referral hospitals.

\begin{tabular}{lccc}
\hline Factors & Category & Frequency & Percentage \\
\hline \multirow{2}{*}{ Smoking habit } & No & 563 & 96.9 \\
& Yes & 18 & 3.1 \\
\hline \multirow{2}{*}{ Daily exercise } & No & 553 & 95.2 \\
& Yes & 28 & 4.8 \\
\hline \multirow{2}{*}{ Alcohol user } & No & 462 & 79.5 \\
& Yes & 119 & 20.5 \\
\hline
\end{tabular}

creatinine greater than $1 \mathrm{mg} / \mathrm{dl}$ had 8.9 times $(\mathrm{AOR}=8.9 ; 95 \%$ CI: 4.40-17.91) higher odds of CKD as compared to serum creatinine less than $1 \mathrm{mg} / \mathrm{dl}$. Patients with dyslipidemia had 3.5 times $(\mathrm{AOR}=3.40$; 95\% CI: 1.56-7.24) $)$ higher odds of CKD as compared to patients with no dyslipidemia (Table 5).

\section{Discussion}

This study was determined the prevalence of CKD among adult hypertensive patients attending the chronic illness follow-up clinic at Northwest Ethiopia referral hospitals and identified associated factors with CKD among adult hypertensive patients.

In the current study, the prevalence of CKD among adult hypertensive patients was $17.6 \%$ (95\% CI: $14.7-20.8 \%$ ). This result was in line with the studies conducted in Jimma (26\%) [22] and Addis Ababa Police Hospital (14.6\%) [11].

However, the finding of this study was lower than the findings of studies conducted in Ghana (46.9\%) [33], Nigeria (38.5\%) [20], Burkina Faso (50.8\%) [34], South China (23\%) [35], Ethiopia (22\%) [11], and South Africa (45.5\%) [36]. This is might be due to the difference in a study subject, study setting, sample size difference, and outcome ascertainment difference. In the previous study conducted in Ghana, the included study participants were aged 16 years and above; whereas, in this study, the included study participants were aged 18 years and above. On the other hand, from the previous study, the settings were polyclinic; whereas, in this study, the study settings are referral hospitals.

This result was higher than studies conducted in Pakistan (12\%) [30]. This difference might be due to differences in a study subject, sample size difference, the difference in the study setting, outcome ascertainment difference, and the contextual difference between the two countries.

According to this study, long duration of hypertension was associated with chronic kidney disease among adult hypertensive patients, which showed that the duration of hypertension greater than 10 years was almost nine times more likely to develop chronic kidney disease as compared to their counterpart. This finding was in agreement with the studies conducted in Ethiopia [11], Jimma [11], and South China [22]. This might be due to persistent increase of blood pressure predispose hypertensive patients to renal vascular nephropathy, which gradually leads to the decrement of glomerular filtration rate and allowing sufficient time to develop other renal diseases; in other way, nephrons in the kidney are supplied with a dense network of blood vessels, and 
TABLE 5: Bivariate and multivariate analyses of hypertensive patients attending at the chronic follow-up clinic at Northwest Ethiopia referral hospitals.

\begin{tabular}{|c|c|c|c|c|}
\hline \multirow{2}{*}{ Variables } & \multicolumn{2}{|c|}{ CKD } & \multirow{2}{*}{ COR } & \multirow{2}{*}{ AOR } \\
\hline & Yes & No & & \\
\hline \multicolumn{5}{|l|}{ SBP } \\
\hline$<140 \mathrm{mmHg}$ & 65 & 452 & 1 & 1 \\
\hline$\geq 140 \mathrm{mmHg}$ & 37 & 27 & $9.529(5.44,16.68)$ & $1.72(0.57,5.21)$ \\
\hline \multicolumn{5}{|l|}{ DBP } \\
\hline$<90 \mathrm{mmHg}$ & 71 & 456 & 1 & 1 \\
\hline$\geq 90 \mathrm{mmHg}$ & 31 & 23 & $8.656(4.77,15.68)$ & $8.65(4.77,15.68)$ \\
\hline \multicolumn{5}{|l|}{ Stage of HTN } \\
\hline Stage I & 9 & 253 & 1 & 1 \\
\hline Stage II & 82 & 206 & $11.19(5.48,22.81)$ & $2.61(1.04,6.50)$ \\
\hline Stage III & 11 & 20 & $15.46(5.73,42.67)$ & $1.18(0.26,5.39)$ \\
\hline \multicolumn{5}{|c|}{ Duration of hypertension } \\
\hline$<10$ years & 86 & 464 & 1 & 1 \\
\hline$\geq 10$ years & 16 & 15 & $5.75(2.74,12.07)$ & $8.81(2.47,31.45)$ \\
\hline \multicolumn{5}{|c|}{ Comorbid disease } \\
\hline No & 91 & 130 & 1 & 1 \\
\hline Yes & 11 & 349 & $22.20(11.51,42.8)$ & $7.0(2.20,22.21)$ \\
\hline \multicolumn{5}{|c|}{ Drugs other than HTN } \\
\hline No & 26 & 347 & 1 & \\
\hline Yes & 76 & 132 & $7.64(4.71,12.52)$ & $0.39(0.14,1.13)$ \\
\hline \multicolumn{5}{|l|}{ Proteinuria } \\
\hline No & 43 & 445 & 1 & 1 \\
\hline Yes & 59 & 34 & $17.95(10.61,30.37)$ & $4.59(2.08,10.12)$ \\
\hline \multicolumn{5}{|l|}{ Dyslipidemia } \\
\hline No & 27 & 409 & 1 & 1 \\
\hline Yes & 75 & 70 & $16.23(9.76,26.96)$ & $3.40(1.56,7.24)$ \\
\hline \multicolumn{5}{|c|}{ Serum creatinine } \\
\hline$<1 \mathrm{mg} / \mathrm{dl}$ & 29 & 434 & 1 & 1 \\
\hline$>1 \mathrm{mg} / \mathrm{dl}$ & 73 & 45 & $24.27(14.31,34.18)$ & $8.88(4.40,17.91)$ \\
\hline
\end{tabular}

the high volume of blood flow through them; overtime uncontrolled high blood pressure can cause arteries around the kidney narrow, weaken, or harden. These damaged arteries are not able to deliver enough blood to the kidney tissue [29].

In this study, presence of comorbid (diabetes and CHF) also associated with CKD among adult hypertensive patients showed that patients with the comorbid disease were 7 times more likely to develop chronic kidney disease as compared to their counterpart. This finding was similar to a study conducted in South Asia [30] and South China [33]. This might be due to diabetes if the patient produces little or no insulin, or if the body cannot use the insulin, the sugar remains in the bloodstream instead of going into the cells. Over time, high levels of sugar in the blood damage tiny blood vessels throughout the body including the filter of the kidney. As more damage occurs to the kidney, more fluid and waste remain in the bloodstream instead of being removed, and in patients with cardiac problems, cardiac output may be low in the glomerular filtration membrane [13].

This study also identified elevated diastolic blood pressure as a significant factor for CKD among adult hypertensive patients, which showed that patients with diastolic blood pressure greater than $90 \mathrm{mmHg}$ were 8.65 times more likely to develop chronic kidney disease as compared to their counterpart. This might be due to patients with increased diastolic blood pressure can cause arteries around the kidney to narrow, weaken, or harden and damages filtration [17].

Based on this study, finding dyslipidemia was associated with CKD among adult hypertensive patients, which showed that patients with dyslipidemia were nearly three and half times more likely to develop chronic kidney disease as compared to their counterpart. This result is similar to a study conducted in Iran [37], Ethiopia [11], and South China [38]. This might be due to dyslipidemia may lace the patients for cardiovascular disease, and besides, it causes cholesterol plaque that can lead to clogging of renal arteries and cuff off blood flow to the kidneys resulting in the loss of kidney function, and also, triglyceride is more associated with cardiovascular risk factors when levels are too high [28].

According to this study, serum creatinine greater than $1 \mathrm{mg} / \mathrm{dl}$ was associated with chronic kidney disease among hypertensive patients, which showed that patients with serum creatinine greater than $1 \mathrm{mg} / \mathrm{dl}$ were 9 times more likely to develop chronic kidney disease among adult hypertensive patients as compared to their counterparts. This result is similar to a study conducted in India [35]. This might be due to as serum creatinine increase. It obstructs the epithelial cells and causes necrotic debris and back leak of filtrate through injured tubular epithelial cells, causing further reduction of the glomerular filtration rate [39]. 
According to this study, proteinuria was associated with chronic kidney disease among adult hypertensive patients, which showed that patients with proteinuria were 4.59 times more likely to develop chronic kidney disease as compared to their counterparts. This finding is similar to a study conducted in Ethiopia [21, 27].

According to this study, stage II HTN was associated with chronic kidney disease, which showed that patients with proteinuria were 2.61 times more likely to develop chronic kidney disease as compared to their counterparts. This finding is similar to a study conducted in India [35]. This might be due to the atherosclerotic, hypertension-related vascular lesions in the kidney primarily affecting the preglomerular arterioles, resulting in ischemic changes in the glomeruli and postglomerular structures. Glomerular injury may also be a consequence of direct damage to the glomerular capillaries due to glomerular hyperperfusion [30]. Since self-reporting was used to this method, it has the disadvantages of recall bias.

The limitation of this study was secondary data, as we could not get potential predictors. It shares also the limitation of the cross-sectional study design.

\section{Conclusion}

In this study, the prevalence of CKD among hypertensive patients was found to be $17.6 \%$. Besides dyslipidemia, proteinuria, comorbid disease, serum creatinine greater than $1 \mathrm{mg} / \mathrm{dl}$, duration of hypertension greater than 10 years, and diastolic blood pressure greater than $90 \mathrm{mmHg}$ are factors associated with the occurrence of chronic kidney disease among hypertensive patients.

\section{Abbreviations}

BMI: $\quad$ Body mass index

BP: $\quad$ Blood pressure

CKD: $\quad$ Chronic kidney disease

CVD: $\quad$ Cardio vascular disease

DM: $\quad$ Diabetes mellitus

DMRH: Debre Markos referral hospital

FHRH: $\quad$ Felege Hiwot referral hospital

HIV/ Human immune virus/acquired immune

AIDS: deficiency syndrome

NSAIDs: Nonsteroidal anti-inflammatory drugs

UOGTRH: University of Gondar teaching referral hospital.

\section{Data Availability}

The data used to support the findings of this study are included within the article, and the dataset analyzed is available from the corresponding author upon request.

\section{Ethical Approval}

Ethical clearance was obtained from the School of Nursing, College Medicine and Health Sciences, University of Gondar. A formal letter indicating the approval was submitted to the University of Gondar Compressive Specialized Hospital Administrative Office, Felege Hiwot Referral Hospital
Administrative Office, and Debre Markos Referral Hospital Administrative Office. Also, it was not revealed to anyone, except for the investigator and supervisor, and was kept in a key and locked system with a computer password.

\section{Consent}

Verbal informed consent was obtained from the study subjects after a purposeful explanation about the objective and the scope of the study.

\section{Conflicts of Interest}

The authors declare that they have no conflicts of interest.

\section{Authors' Contributions}

AH designed the study, developed the proposal, participated in the data collection, performed analysis, and drafted the manuscript. MAT, HSM, and CDA approved the proposal with revisions, participated in data analysis, and revised subsequent drafts of the manuscript. All authors read and approved the final manuscript.

\section{Acknowledgments}

The authors would like to express their gratitude to the University of Gondar for the approval of the ethical clearance. The authors would like to thank the respective referral hospitals administration offices for their permission letter and data collectors and supervisors for their commitment and the study participants for their valuable information.

\section{References}

[1] J. T. DiPiro, R. L. Talbert, G. C. Yee, G. R. Matzke, B. G. Wells, and L. M. Posey, Pharmacotherapy: A Pathophysiologic Approach, McGraw Hill, New York, NY, USA, 2014.

[2] A. Whaley-Connell, J. R. Sowers, P. A. McCullough et al., "Diabetes mellitus and CKD awareness: the kidney early evaluation program (KEEP) and national health and nutrition examination survey (NHANES)," American Journal of Kidney Diseases, vol. 53, no. 4, pp. S11-S21, 2009.

[3] G. Eknoyan, N. Lameire, K. Eckardt et al., "KDIGO 2012 clinical practice guideline for the evaluation and management of chronic kidney disease," Kidney International, vol. 3, no. 1, pp. 5-14, 2013.

[4] A. Levin and M. Rocco, "KDOQI clinical practice guidelines and clinical practice recommendations for diabetes and chronic kidney disease," American Journal of Kidney Diseases, vol. 49, no. 2, pp. S12-S154, 2007.

[5] K. P. Wanigasuriya, R. J. Peiris-John, and R. Wickremasinghe, "Chronic kidney disease of unknown aetiology in Sri Lanka: is cadmium a likely cause?" BMC Nephrology, vol. 12, no. 1, p. 32, 2011.

[6] D. Bahrey, G. Gebremedhn, T. Mariye et al., "Prevalence and associated factors of chronic kidney disease among adult hypertensive patients in Tigray teaching hospitals: a crosssectional study," BMC Research Notes, vol. 12, no. 1, p. 562, 2019. 
[7] A. M. El Nahas and A. K. Bello, "Chronic kidney disease: the global challenge,” The Lancet, vol. 365, no. 9456, pp. 331-340, 2005.

[8] CDC, Chronic Kidney Disease Basics, Centers for Disease Control and Prevention, Atlanta, GA, USA, 2021, https:// www.cdc.gov/kidneydisease/index.html.

[9] S. M. Newton, A. J. Brent, S. Anderson, E. Whittaker, and B. Kampmann, "Paediatric tuberculosis," The Lancet Infectious Diseases, vol. 8, no. 8, pp. 498-510, 2008.

[10] N. R. Hill, S. T. Fatoba, J. L. Oke et al., "Global prevalence of chronic kidney disease-a systematic review and metaanalysis," PLoS One, vol. 11, no. 7, Article ID e0158765, 2016.

[11] K. Kumela Goro, A. Desalegn Wolide, F. Kerga Dibaba et al., "Patient awareness, prevalence, and risk factors of chronic kidney disease among diabetes mellitus and hypertensive patients at Jimma university medical center, Ethiopia," BioMed Research International, vol. 2019, Article ID 2383508, 8 pages, 2019.

[12] WHONdNcRopddtNiE, 2018.

[13] E. Di Angelantonio, R. Chowdhury, N. Sarwar, T. Aspelund, J. Danesh, and V. Gudnason, "Chronic kidney disease and risk of major cardiovascular disease and non-vascular mortality: prospective population based cohort study," BMJ, vol. 341, Article ID c4986, 2010.

[14] J. Bommer, "Prevalence and socio-economic aspects of chronic kidney disease," Nephrology Dialysis Transplantation, vol. 17, no. suppl_11, pp. 8-12, 2002.

[15] World Health Organization, Nutritional Anaemias: Tools for Effective Prevention and Control, WHO, Geneva, Switzerland, 2017.

[16] GBD 2013 Mortality and Causes of Death Collaborators, "Global, regional, and national age-sex specific all-cause and cause-specific mortality for 240 causes of death, 1990-2013: a systematic analysis for the global burden of disease study 2013," The Lancet, vol. 385, no. 9963, pp. 117-171, 2015.

[17] J. Coresh, "Update on the burden of CKD," Journal of the American Society of Nephrology, vol. 28, no. 4, pp. 1020-1022, 2017.

[18] Ethiopia'S ASP and PIF IF, Federal Democratic Republic of Ethiopia Ministry of Agriculture and Rural Development, Ministry of Agriculture, Addis Ababa, Ethiopia, 2010.

[19] World Health Organization, Weekly Epidemiological Record, WHO, Geneva, Switzerland, 2004.

[20] M. A. S. Mbengue, M. Sarr, A. Faye et al., "Determinants of complete immunization among senegalese children aged 12-23 months: evidence from the demographic and health survey," BMC Public Health, vol. 17, no. 1, p. 630, 2017.

[21] P. E. Fine, I. A. Carneiro, J. B. Milstien, C. J. Clements, and World Health Organization, Issues Relating to the Use of BCG in Immunization Programmes: A Discussion Document, World Health Organization, Geneva, Switzerland, 1999.

[22] B. O. Olusanya, "Pattern and determinants of BCG immunisation delays in a sub-Saharan African community," Health Research Policy and Systems, vol. 8, no. 1, 2010.

[23] K. Krivoruchko, Empirical Bayesian Kriging. ArcUser, Fall 2012, Esri, West Redlands, CA, USA, 2012.

[24] S. Naish and S. Tong, "Hot spot detection and spatio-temporal dynamics of dengue in Queensland, Australia," in Proceedings of the ISPRS Technical Commission VIII Symposium, International Society of Photogrammetry and Remote Sensing (ISPRS), Berlin, Germany, June 2014.

[25] L. Mitas and H. Mitasova, "Spatial interpolation," Geographical Information Systems: Principles, Techniques, Management and Applications, vol. 1, no. 2, 1999.
[26] A. Roy, M. Eisenhut, R. J. Harris et al., "Effect of BCG vaccination against mycobacterium tuberculosis infection in children: systematic review and meta-analysis," BMJ, vol. 349, p. g4643, 2014.

[27] M. L. Berendsen, S. W. Van Gijzel, J. Smits et al., "BCG vaccination is associated with reduced malaria prevalence in children under the age of 5 years in sub-Saharan Africa," BMJ Global Health, vol. 4, no. 6, Article ID e001862, 2019.

[28] G. Biesenbach, P. Grafinger, O. Janko, and J. Zazgornik, "Influence of cigarette-smoking on the progression of clinical diabetic nephropathy in type 2 diabetic patients," Clinical Nephrology, vol. 48, no. 3, pp. 146-150, 1997.

[29] A. C. Webster, E. V. Nagler, R. L. Morton, and P. Masson, "Chronic kidney disease," The Lancet, vol. 389, no. 10075, pp. 1238-1252, 2017.

[30] L. Anselin, S. Sridharan, and S. Gholston, "Using exploratory spatial data analysis to leverage social indicator databases: the discovery of interesting patterns," Social Indicators Research, vol. 82, no. 2, pp. 287-309, 2007.

[31] R. El Edelbi, S. Lindemalm, and S. Eksborg, "Estimation of body surface area in various childhood ages - validation of the mosteller formula," Acta Paediatrica, vol. 101, no. 5, pp. 540-544, 2012.

[32] S. Asfaw, F. Enquselassie, Y. Tefera, M. Gizaw, S. Wakuma, and M. Woldemariam, "Determinants of chronic respiratory symptoms among pharmaceutical factory workers," Journal of Tropical Medicine, vol. 2018, Article ID 3815689, 10 pages, 2018.

[33] S. Hailu, A. Astatkie, K. A. Johansson, and B. Lindtjørn, "Low immunization coverage in Wonago district, southern Ethiopia: a community-based cross-sectional study," PLoS One, vol. 14, no. 7, Article ID e0220144, 2019.

[34] A. Lengani, A. Samadoulougou, and M. Cissé, "Characteristics of renal disease in hypertensive morbidities in adults in Burkina Faso," Archives des Maladies du Coeur et des Vaisseaux, vol. 93, pp. 1053-1057, 2000.

[35] L. C. Zulu, E. Kalipeni, and E. Johannes, "Analyzing spatial clustering and the spatiotemporal nature and trends of HIV/ AIDS prevalence using GIS: the case of Malawi, 1994-2010," BMC Infectious Diseases, vol. 14, no. 1, p. 285, 2014.

[36] L. S. Da Silva, R. M. M. Cotta, T. R. Moreira et al., "Hidden prevalence of chronic kidney disease in hypertensive patients: the strategic role of primary health care," Public Health, vol. 140, pp. 250-257, 2016.

[37] T. N. Jilani, A. Avula, A. Z. Gondal, and A. H. Siddiqui, "Active tuberculosis," StatPearls, StatPearls Publishing, Treasure Land, FL, USA, 2019.

[38] M. Ahmed, M. Demissie, A. Worku, A. Abrha, and Y. Berhane, "Socio-cultural factors favoring home delivery in Afar pastoral community, northeast Ethiopia: a qualitative study," Reproductive Health, vol. 16, no. 1, pp. 171-179, 2019.

[39] J. Segura, C. Campo, P. Gil et al., "Development of chronic kidney disease and cardiovascular prognosis in essential hypertensive patients," Journal of the American Society of Nephrology, vol. 15, no. 6, pp. 1616-1622, 2004. 